\title{
Spectroscopy of HD 86222 - a quintuple system with an eclipsing component ${ }^{\star, \star \star}$
}

\author{
W. Dimitrov ${ }^{1}$, M. Fagas ${ }^{1}$, K. Kamiński ${ }^{1}$, D. Kolev ${ }^{2}$, T. Kwiatkowski ${ }^{1}$, \\ K. Bạkowska ${ }^{1,3}$, A. Rożek ${ }^{1,4}$, P. Bartczak ${ }^{1}$, W. Borczyk ${ }^{1}$, and A. Schwarzenberg-Czerny 1,3 \\ 1 Astronomical Observatory Institute, Faculty of Physics, A. Mickiewicz University, Słoneczna 36, 60-286 Poznań, Poland \\ e-mail: dimitrov@amu.edu.pl \\ 2 National Astronomical Observatory Rozhen, PO Box 136, 4700 Smolyan, Bulgaria \\ 3 Nicolaus Copernicus Astronomical Center, Polish Academy of Sciences, Bartycka 18, 00-716 Warszawa, Poland \\ ${ }^{4}$ Centre for Astrophysics and Planetary Science, University of Kent, Canterbury, Kent, CT2 7NH, UK
}

Received 12 February 2013 / Accepted 22 January 2014

\section{ABSTRACT}

\begin{abstract}
We present spectroscopic and photometric analysis of the quintuple star HD 86222 with an eclipsing component. Until now three visual components A, B, and C of this multiple star were known. Four components in the A/B pair were detected during the examination of the cross correlation functions obtained from the spectra. We noticed that the visual components A and B, separated by 0 .'5, are in fact two binary stars - one eclipsing pair and one spectroscopic system. The pair with higher radial velocity amplitude corresponds to the eclipsing period. Photometric and spectroscopic observations of the eclipsing binary enable us to obtain the Wilson-Devinney model. The masses of this pair are $M_{1}=1.29 \pm 0.09 M_{\odot}$ and $M_{2}=1.33 \pm 0.09 M_{\odot}$, respectively. The radii, slightly higher than for the main sequence stars, are $R_{1}=1.35 \pm 0.01 R_{\odot}$ and $R_{2}=1.36 \pm 0.01 R_{\odot}$, respectively. The main parameters of the spectroscopic binary are also estimated, but they must be confirmed by future observations because of the uncertainty of the period. The farthest star named C, is 15 arc seconds from the main A and B components. Assuming that this object is gravitationally connected with the A and B binary pairs, HD 86222 has at least five components.
\end{abstract}

Key words. binaries: close - binaries: eclipsing - stars: individual: HD 86222

\section{Introduction}

Eclipsing binary stars are important for determining the absolute parameters of the stars like masses, radii, and distance. According to Chambliss (1992), about 80 eclipsing binaries are components of multiple star systems, most of them triple. Chamblis mentions only three or four probable quintuple systems, some of which have visual components. Zasche et al. (2009) presents a catalog of eclipsing binaries (EBs) that are components of the spatialy resolved pairs. The system HD 86222 is a rare quintuple hierarchical system with an eclipsing component. Three of the components are visually resolved which enables future determination of the visual orbits. Recently, a new object with a similar hierarchy has been discovered: the eclipsing quintuple system V994 Herculi (Lee et al. 2008; Zasche \& Uhlař 2013).

The multiple star HD 86222 is listed in the CCDM catalog (Dommanget \& Nys 1994) as a triple visually resolved system. The equatorial coordinates of the system are $\alpha=09^{\mathrm{h}} 57^{\mathrm{m}} 06.6^{\mathrm{s}}$ and $\delta=-01^{\circ} 20^{\prime} 38^{\prime \prime}$ (FK5). The main components $\mathrm{A}$ and $\mathrm{B}$ are separated by 0.5 and they have brightness 9.5 and 9.8 ( $V$ ) magnitude, respectively. The position angle of the A and B components is $157^{\circ}$ based on measurements from 1943. The third component $\mathrm{C}$ is also listed in the CCDM catalog with a

* Based on NAO Rozhen and PST1 spectroscopy and ASAS photometry.

$\star \star$ The spectra are only available at the CDS via anonymous ftp to cdsarc.u-strasbg. fr (130.79.128.5) or via

http://cdsarc.u-strasbg.fr/viz-bin/qcat?J/A+A/564/A26 lower brightness of $14^{\mathrm{m}} .0$ and higher separation $15^{\prime \prime} .0$ than previously described stars of the HD 86222 system. The position angle of $13^{\circ}$ was measured in 1908. Other position angle measurements were published by Heintz (1975) and Couteau (1967). The most up-to-date measurements are in the Washington Visual Double Star Catalog (Mason et al. 2001), where separation between $\mathrm{A}$ and $\mathrm{B}$ is $0 .^{\prime} 50$ and $\mathrm{PA}=183^{\circ}(2004)$ and separation between $\mathrm{C}$ and $\mathrm{A} / \mathrm{B}$ is $17^{\prime \prime}$. $40, \mathrm{PA}=3^{\circ}$ (2000).

The system is listed in the Henry Draper Catalogue as an F5 spectral type. The color index $B-V=0.45$, was published in the Tycho Catalogue, and its value corresponds to the temperature of $6600 \mathrm{~K}$ (spectral type F5). The object was observed by ASAS (All Sky Automated Survey, Pojmanski 1997) and classified as ASAS 095706-0120.7. During this survey, eclipses with similar depth and with the amplitude of 0.15 were detected. The period of the object was also calculated; it is very close to one day (0d.987) which cause difficulties in observation campaigns.

The original objective for observing this star was to get its radial velocity curve and, using the ASAS photometry, to derive its model. After the analysis of the spectroscopic data it appeared that the HD 86222 system is more complex than previously assumed and that it can be considered a quintuple star.

\section{Data}

\subsection{Spectroscopy}

The first spectra of HD 86222 AB were obtained on 8 January 2004 with the $2 \mathrm{~m}$ RCC telescope at NAO Rozhen, equiped with 

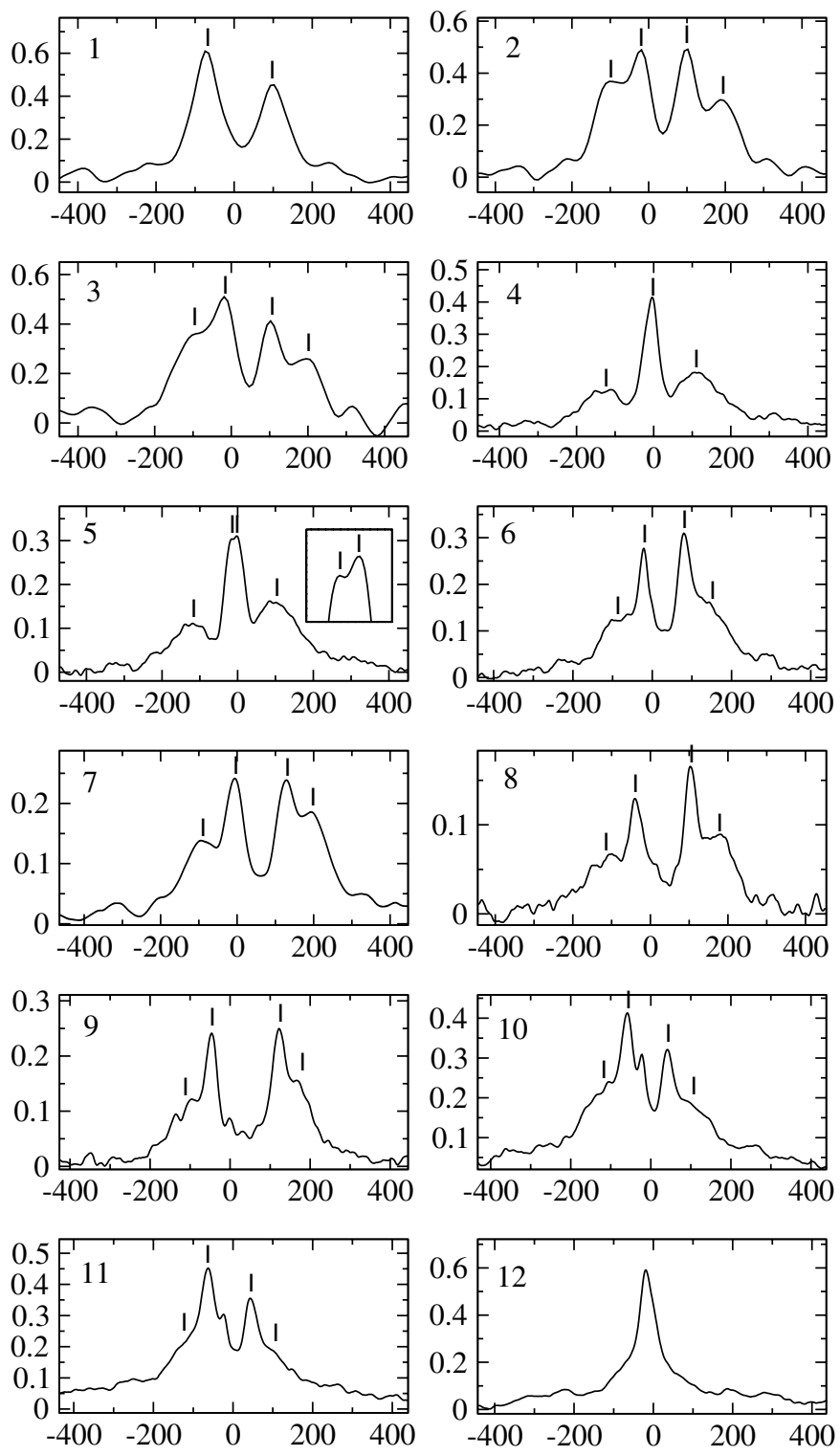

Fig. 1. Cross correlation function for all spectra listed in Table 1. Numbers of the plots correspond to the Table 1 order, $x$-axis represents the observed velocities, and $y$-axis the correlation coefficient. The measured velocities are marked. Additionally, one CCF plot (12) is presented with all four peaks blended. First three plots are from the Rozhen spectra, the rest are from PST 1.

a coudé spectrograph. The spectral range was $200 \AA$ near the MgI triplet at $5175 \AA$ with a dispersion of $0.2 \AA /$ pix. The exposure times were $1200 \mathrm{~s}$. The cross correlation function (CCF) in some phases revealed four clear peaks.

To confirm the detection of the four components we started observations on the new $0.4 \mathrm{~m}$ Poznan Spectroscopic Telescope 1 (PST 1) equipped with fiber fed echelle spectrograph (detailed description in Baranowski et al. 2009). Its high photon efficiency allows RV measurements for objects as faint as $11 \mathrm{mag}$. The detector is a five-stage Peltier/liquid cooled $2 \mathrm{~K} \times 2 \mathrm{~K}$ Andor $\mathrm{CCD}$ working at $-90^{\circ} \mathrm{C}$. The $\mathrm{CCD}$ camera covers 64 echelle orders with spectral range 4500-9250 $\AA$. The exposure times were $1800 \mathrm{~s}$. The PST 1 spectra also presents in some phases four peaks in CCF (Fig. 1). DY Lyncis is another multiple object with the eclipsing component observed with this telescope for which we found a third component spectroscopically (Sekalska et al. 2010).
Table 1. Radial velocities of the four components of HD 86222 AB.

\begin{tabular}{lcccc}
\hline \hline $\begin{array}{l}\text { HJD } \\
2450000 .+\end{array}$ & $\begin{array}{c}\text { RV EBa } \\
\mathrm{km} \mathrm{s}^{-1}\end{array}$ & $\begin{array}{c}\text { RV EBb } \\
\mathrm{km} \mathrm{s}^{-1}\end{array}$ & $\begin{array}{c}\text { RV SBa } \\
\mathrm{km} \mathrm{s}^{-1}\end{array}$ & $\begin{array}{c}\text { RV SBb } \\
\mathrm{km} \mathrm{s}^{-1}\end{array}$ \\
\hline NAO Rozhen \\
\hline 3013.585073 & -45.6 & 110.9 & -45.6 & 110.9 \\
3096.328784 & -120.1 & 174.8 & 79.2 & -38.3 \\
\hline \multicolumn{5}{c}{ PST 1 } \\
\hline 4493.562774 & 143.3 & -94.9 & 27.0 & - \\
4493.588979 & 133.0 & -88.7 & 9.4 & 36.0 \\
4571.366165 & 145.2 & -93.2 & 78.5 & -20.6 \\
4581.324491 & 173.1 & -119.1 & -30.8 & 100.0 \\
4583.317248 & 168.8 & -120.1 & -41.5 & 98.6 \\
4584.372909 & 174.2 & -111.7 & -50.2 & 116.9 \\
4843.525536 & -80.7 & 146.4 & -17.9 & 81.5 \\
4843.552169 & -77.0 & 138.9 & -23.2 & 84.9 \\
\hline
\end{tabular}

The typical signal-to-noise ratio is about 25 for PST1 and about 100 for NAO Rozhen spectra. The PST1 spectra are more noisy but they cover about 24 times wider range.

The bright pairs A and B are separated only by 0.'5 so our spectra contains light from both of them. Because ASAS data reveal regular eclipses, one of the $\mathrm{A}$ and $\mathrm{B}$ components must be an eclipsing pair. The other component is a spectroscopic binary. Untill now we have not been able to identify which pair corresponds to the A and B components (see Sect. 4). The easiest way to identify the components is to observe them separately, but with a very small angular separation of 0.5 , that requires very good seeing or adaptive optics. In the text below we will use the name $\mathrm{EBa} \& \mathrm{EBb}$ for an eclipsing pair and SB for the spectroscopic pair.

The CCF in phase 0.41 (Fig. 1, plot 1 ) shows the moment of passage of the peaks of SB and EB. For this spectrum we only have two CCF peaks. Our team have more spectra around this phase, which cannot be measured because of the high blending of the peaks. Nevertheless, these spectra enabled us to determine the moment of the equal velocities.

Data reduction and radial velocity measurements were provided by IRAF $^{1}$ package tasks. We used DCR software for cosmic ray removal (Pych 2004).

\subsection{Photometry}

For the photometric analysis of the system we used the ASAS photometry (Pojmanski 1997). The $I$-band set of 912 measurements was obtained during the period from 19 October 1998 to 18 January 2000 and the $369 \mathrm{~V}$ magnitudes were observed from 10 December 2000 to 5 November 2008. The second set were noisier (see Fig. 2) but were included in the analysis because they extend the time span covered by the observations to about 10 years. This increased the accuracy of the orbital period determination. Some outstanding points in the lightcurves (nine in $I$ and four in $V$ ) were rejected.

Based on both $I$ and $V$ data, we derived the following ephemeris for the eclipsing pair:

$\min I=$ HJD $2451234.5236(65)+0.987045(5) \cdot E$.

1 IRAF is distributed by the National Optical Astronomy Observatory, which is operated by the Association of Universities for Research in Astronomy, Inc., under a cooperative agreement with the National Science Foundation. 
W. Dimitrov et al.: Spectroscopy of a quintuple system HD 86222

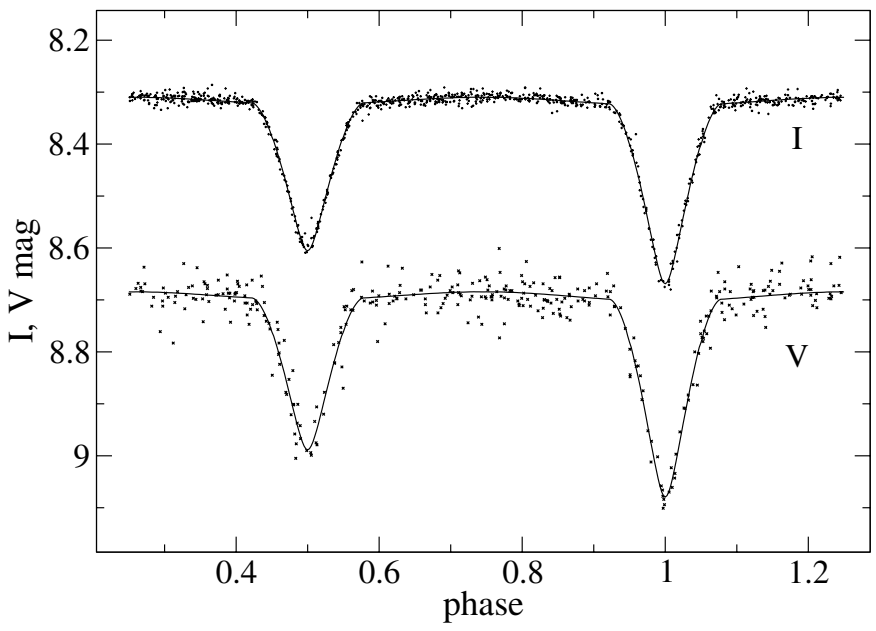

Fig. 2. Synthetic light curves for HD 86222 compared with ASAS $I$ and $V$-band data.

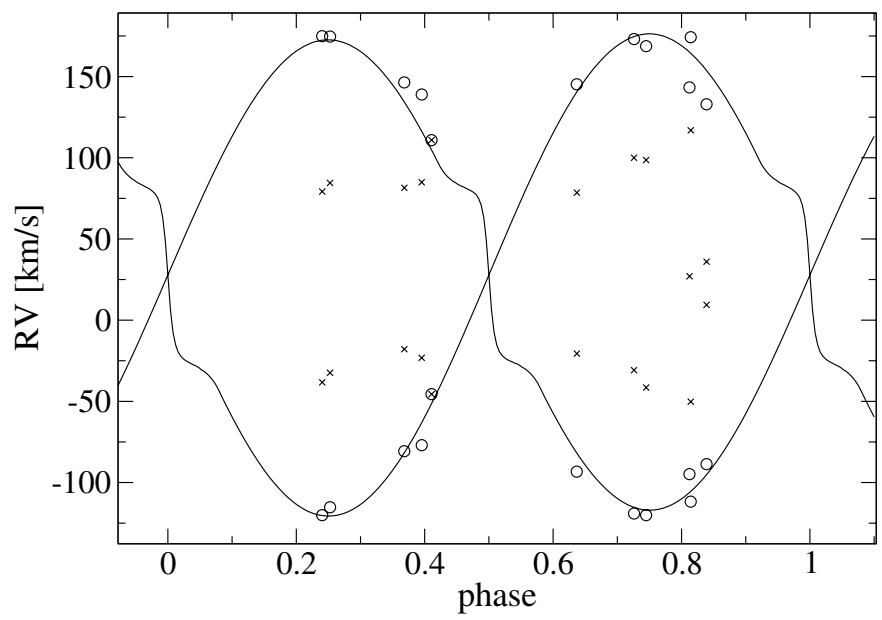

Fig. 3. Radial velocity curve for the eclipsing pair. Circles represent the velocities of the EB components and "x" signs are the components of the spectroscopic pair.

The ASAS photometry is contaminated by the farthest component $\mathrm{C}$. The ratio of the $\mathrm{C}$ and $\mathrm{A} / \mathrm{B}$ fluxes is about $10^{-2}$.

\section{Model of the system}

\subsection{The eclipsing pair}

After phasing of the RV measurements with the photometric period we found that the eclipses correspond to the pair of $\mathrm{CCF}$ peaks with higher velocity and lower height. In the second panel of Fig. 1 the two higher peaks are connected with the spectroscopic binary and the outer pair with the eclipsing binary. We used the PHOEBE code (Prša \& Zwitter 2005) based on the Wilson \& Devinney (1971) method for our radial velocities (Fig. 3) and ASAS photometric measurements (Fig. 2). We treated the spectroscopic pair as a constant third light. The obtained model (Table 2) of the binary showed two similar components with masses about $1.3 M_{\odot}$ close to the main sequence and slightly evolved.

The distance to the eclipsing binary can be derived with the photometric parallax method. As we cannot identify the components, because of their proximity and similar brightness, we consider two possible cases. First, the eclipsing pair is the brighter component A of the system, and second, the EB is the
Table 2. Preliminary parameters of the eclipsing pair obtained with the Wilson-Devinney method.

\begin{tabular}{lcc}
\hline \hline Parameter & \multicolumn{2}{c}{ Eclipsing pair } \\
& EBa & $\mathrm{EBb}$ \\
\hline Period & $0.987045(5)$ \\
$i$ & $86.7 \pm 0.1$ \\
$q$ & $1.0243 \pm 0.0008$ \\
$a\left(R_{\odot}\right)$ & $5.75 \pm 0.13$ \\
$V_{\gamma}\left(\mathrm{km} \mathrm{s}^{-1}\right)$ & $27.8 \pm 2.9$ \\
$\Omega$ & \\
$\Omega$ & $5.32 \pm 0.03$ & $5.37 \pm 0.03$ \\
$l_{I}$ & $0.316 \pm 0.004$ & $0.266 \pm 0.003$ \\
$l_{V}$ & $0.336 \pm 0.006$ & $0.266 \pm 0.005$ \\
$l_{3}(I)$ & $0.419 \pm 0.001$ \\
$l_{3}(V)$ & $0.398 \pm 0.016$ \\
\multicolumn{3}{c}{} \\
Mass $\left(M_{\odot}\right)$ & $1.29 \pm 0.09$ \\
Radii $\left(R_{\odot}\right)$ & $1.35 \pm 0.01$ & $1.33 \pm 0.09$ \\
$T_{\text {eff }}(\mathrm{K})$ & 6440 fixed & $6160 \pm 0.01$ \\
\hline
\end{tabular}

Notes. The spectroscopic pair is treated as a third light $\left(l_{3}\right)$. Errors (except period) are calculated with Monte Carlo simulation.

fainter component B. The obtained distances are $189 \pm 23 \mathrm{pc}$ and $217 \pm 26 \mathrm{pc}$, respectively. We have no direct information on the individual colors of the four components owing to the mixing of their light. Therefore, we assume the temperature of the main EB component to be $6440 \mathrm{~K}$ corresponding to the temperature of the MS star with the same mass. The temperature of the secondary EB component and its error comes from the WilsonDevinney model.

Because of the small separation and distortion of the components we assumed circular orbit and synchronous rotation of both stars. We applied albedo and gravity brightening coeficents for convective envelope, $A_{12}=0.5$ and $g_{12}=0.32$, respectively. For the calculation of the limb darkening effect we adopt van Hamme (1993) tables and the logarithmic law.

The systemic velocities of EB and SB pairs are almost equal, which we can expect from bonded gravitationally but well separated stars.

\subsection{The spectroscopic pair}

The main problem for the spectroscopic binary is to find the orbital period because of the lack of the eclipses. We used both photometric and spectroscopic data. We searched for ellipsoidal variations in I-band data after subtraction of the synthetic EB curve from the data. We found some periodicities, but they were rejected by the spectroscopic data. In the RV data we found a huge number of possible periods, but after using additional information about the height of the CCF peaks (component one is more luminous) we only obtained one result fitting to the data $P=1.053$, which is very close to the EB period. We used the G. Maciejewski period search software based on the Schwarzenberg-Czerny (1996) method.

We have two subsequent spectra close to the phase with minimal radial velocities (Fig. 4). The first presents a single peak; the next is slightly dissociated, which means that we have the moment of the minimal RVs, but the period must be confirmed by future observations. Based on the RV data we obtained the ephemeris

$\min \mathrm{RV}=$ HJD $2454493.5(63)+1.05282(4) \cdot E$. 

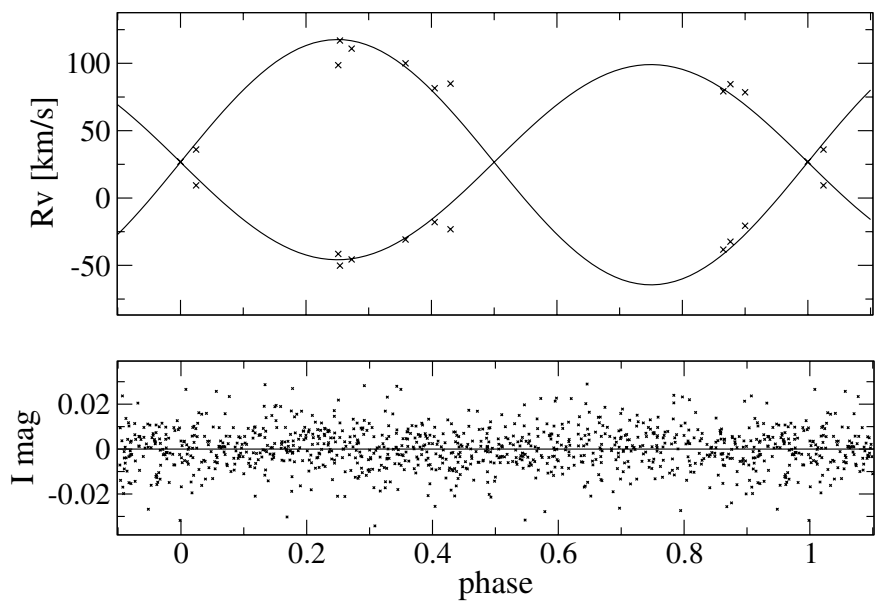

Fig. 4. Radial velocity and light curves for the spectroscopic pair. The curves are phased with our best-fit period (Eq. (2)). Light curve presents the I data after subtraction of the eclipsing binary variability.

Table 3. Preliminary results and formal errors for the spectroscopic pair.

\begin{tabular}{lc}
\hline \hline Parameter & Value \\
\hline Period & $1.05282(4)$ \\
$q$ & $0.884 \pm 0.060$ \\
$a \cdot \sin i\left(R_{\odot}\right)$ & $3.39 \pm 0.11$ \\
$m_{1} \cdot \sin ^{3} i\left(M_{\odot}\right)$ & $0.251 \pm 0.093$ \\
$m_{2} \cdot \sin ^{3} i\left(M_{\odot}\right)$ & $0.222 \pm 0.083$ \\
$V_{\gamma}\left(\mathrm{km} \mathrm{s}^{-1}\right)$ & $27.7 \pm 2.0$ \\
\hline
\end{tabular}

The ephemeris presents the moments of minimum radial velocity, i.e., inferior conjunction. Photometric data revealed no eclipses for the SB, which means that we have no direct information about the orbital inclination of the spectroscopic pair. The spectroscopic curve gives us only the lower limit of mass of the SB components (Table 3). Furthermore, we know that SB and $\mathrm{EB}$, which are the two main visual components of the HD 86222 system, have a similar brightness. This luminosity requires an $\mathrm{SB}$ orbital inclination about $35^{\circ}$.

Assuming that both binaries are at the same distance and taking into account the relative brightness from the light curve solution and SB mass ratio from spectroscopy, we can estimate the masses of the spectroscopic pair components. We obtained $M_{1} \simeq 1.24 M_{\odot}$ and $M_{2} \simeq 1.10 M_{\odot}$ and the corresponding orbital inclination about $36^{\circ} .0$. Calculating the semi-major axis for SB we obtained $5.8 R_{\odot}$, which is very close to the value for the EB orbit.

\subsection{Component $C$}

During the last century, according to the Washington Visual Double Star Catalog, the position angle of component $\mathrm{C}$ has changed by $12^{\circ}$. Assuming the circular orbit perpendicular to the line of sight, we can make a rough estimation of the orbital period of component $\mathrm{C}$ to be about $3 \times 10^{3}$ years. Taking into account the angular separation and the distance, the size of the semi-major axis is about $3000 \mathrm{AU}$. The distance to the system and component brightness corresponds to the $\mathrm{M}$ spectral type star.

\section{Discussion and conclusions}

The visual components A and B of HD 86222 are of similar brightness and this makes it difficult to determine which one is the eclipsing pair. The argument for the eclipsing binary being the brighter one (A) is that the WD model suggests the third light (the spectroscopic binary) is less than $50 \%$ of the light of the A/B system. This is observed in both filters. The argument for the opposite case, in which the spectroscopic binary is the brighter one, is based on the fact that the peaks in the cross correlation function corresponding to the SB components have slightly higher surfaces. We measured the surfaces under the Gaussian functions fitted to the CCF for the four spectra with well-resolved peaks. We found out that the ratio of SB to EB surfaces is $1.2 \pm 0.2$. This value sugests that SB is more luminous.

To solve the problem of the identification of the components we can use different techniques (Gorda 1994). A first solution could be measuring the brightness of the A and B components during the eclipse and after the eclipse. This requires a very good seeing or adaptive optics. Alternatively, we can measure the shift of the photocentrum during the minimum.

Based on the position angle changes, the angular separation, and distance we can make a rough estimate of the orbital period and the semi-major axis of the mutual orbit of the A and B components. The former would be about 900 years, and the latter about $100 \mathrm{AU}$.

The main result of our spectroscopic observations of HD 86222 is the detection of new components of this system. It appears that both A and B stars are pairs. Additionaly, we derived a preliminary solution for the eclipsing pair. We are planning to continue observations of HD 86222 to confirm the SB period, and to find out which component, A or B, is the eclipsing one.

Acknowledgements. This work was supported by the Polish National Science Centre through grant UMO-2011/01/D/ST9/00427.

\section{References}

Baranowski, R., Smolec, R., Dimitrov, W., et al. 2009, MNRAS, 396, 2194 Chambliss, C. R. 1992, PASP, 104, 663

Couteau, P. 1967, Journal des Observateurs, 50, 41

Dommanget, J., \& Nys, O. 1994, Communications de l'Observatoire Royal de Belgique, 115

Gorda, S. Y. 1994, Astron. Lett., 20, 446

Heintz, W. D. 1975, ApJS, 29, 315

Lee, C.-U., Kim, S.-L., Lee, J. W., et al. 2008, MNRAS, 389, 1630

Mason, B. D., Wycoff, G. L., Hartkopf, W. I., Douglass, G. G., \& Worley, C. E. 2001, AJ, 122, 3466

Pojmanski, G. 1997, Acta Astron., 47, 467

Prša, A., \& Zwitter, T. 2005, ApJ, 628, 426

Pych, W. 2004, PASP, 116, 148

Schwarzenberg-Czerny, A. 1996, ApJ, 460, L107

Sekalska, J., Dimitrov, W., Fagas, M., et al. 2010, IBVS, 5954, 1

van Hamme, W. 1993, AJ, 106, 2096

Wilson, R. E., \& Devinney, E. J. 1971, ApJ, 166, 605

Zasche, P., \& Uhlař, R. 2013, MNRAS, 570

Zasche, P., Wolf, M., Hartkopf, W. I., et al. 2009, AJ, 138, 664 